\title{
Aux sources de la relecture de l'histoire de la Révolution française par François Furet
}

La lutte des intellectuels français contre le «totalitarisme » et ses effets sur l'histoire de l'historiographie de la Révolution française

Michael Scott Christofferson, Jacques Guilhaumou et Julien Louvrier

\section{(2) OpenEdition}

Journals

Édition électronique

URL : https://journals.openedition.org/ahrf/11775

DOI : $10.4000 / a h r f .11775$

ISSN : 1952-403X

Éditeur :

Armand Colin, Société des études robespierristes

Édition imprimée

Date de publication : 1 juin 2010

Pagination : 227-238

ISBN : 978-2-200-92632-8

ISSN : 0003-4436

Référence électronique

Michael Scott Christofferson, Jacques Guilhaumou et Julien Louvrier, « Aux sources de la relecture de I'histoire de la Révolution française par François Furet », Annales historiques de la Révolution française [En ligne], 360 | avril-juin 2010, mis en ligne le 01 juin 2013, consulté le 23 avril 2022. URL : http:// journals.openedition.org/ahrf/11775; DOI : https://doi.org/10.4000/ahrf.11775 


\title{
AUX SOURCES DE LA RELECTURE DE L'HISTOIRE DE LA RÉVOLUTION FRANÇAISE PAR FRANÇOIS FURET
}

\author{
LA LUTTE DES INTELLECTUELS FRANÇAIS CONTRE \\ LE «TOTALITARISME » ET SES EFFETS SUR L'HISTOIRE \\ DE L'HISTORIOGRAPHIE DE LA RÉVOLUTION FRANÇAISE
}

A propos de Michael Scott Christofferson, Les intellectuels contre la gauche. L'idéologie antitotalitaire en France (1968-1981), Marseille, Agone, 2009, XIV-445p.

\author{
Michael Scott CHRISTOFFERSON \\ Jacques GUILHAUMOU \\ Julien LOUVRIER
}

French Intellectuals Against the Left, le livre consacré en 2004 par I'historien américain Michael Scott Christofferson à I'histoire du « momentantitotalitaire »enFrance,arécemmentfaitl'objetd'unetraduction en français' ${ }^{1}$. Dans ce livre, issu d'une thèse de doctorat menée sous la direction de Robert Paxton, Christofferson part du constat troublant que la critique du «totalitarisme » s'est développée en France dans les années 1970 au moment où en Allemagne et aux Etats-Unis, là même où leconceptavaitinitialementprisforme, sonopérabilitéétaitsérieusement remise en question. Ayant identifié dans les réactions consécutives à la

(1) Michael Scott Christofferson, French Intellectuals against the Left. The Antitotalitarian Moment of the 1970s, New York, Oxford, Berghahn Books, 2004, X-294p. Les intellectuels contre la gauche. L'idéologie antitotalitaire en France (1968-1981), traduit de l'anglais par André Merlot, Marseille, Agone, 2009, XIV-445p. La traduction française du livre fera l'objet d'un compte rendu dans un prochain numéro de la revue. 
publication du livre de Soljenitsyne, L'Archipel du Goulag, le « mythe des origines del'antitotalitarisme », il en propose une déconstruction méticuleuse. L'idée d'une prise de conscience, ou d'un « effet Goulag » qu'aurait provoqué la lecture de Soljenitsyne relève, explique-t-il, de l'escroquerie intellectuelle, car tous les connaisseurs de l'Union soviétique s'accordent à dire que le livre ne comportait aucune révélation. Cen'est pas l'existence du Goulag qui agite les intellectuels parisiens au milieu des années 1970, mais l'inquiétude de voir les communistes français arriver au pouvoir dans le cadre de l'Union de la gauche. Débarrassé de ses dimensions humanitaires,l'antitotalitarismeapparaîtalorscommeuninstrumentidéologique franco-françaisbrandipardesintellectuelsdelagauchenon-communiste, parfois d'ex-communistes, prêts à tout pour laminer l'influence du PCF dont ils dénoncent l'hégémonie idéologique à gauche. Mais comment accréditer l'idée que la France risque de basculer dans le totalitarisme en cas de victoire électorale du Parti socialiste et de ses alliés communistes? C'est là qu'intervient François Furet, nous explique Michael Christofferson qui consacre le dernier chapitre de son enquête à l'enjeu constitué par l'écriture de l'histoire de la Révolution française et sa relecture par Furet, l'ancien communiste. En appliquant à la Révolution française la critique du totalitarisme, en situant les origines du totalitarisme dans la Révolution française - qualifiée de « matrice » du totalitarisme - François Furet donne de la crédibilité à l'idée selon laquelle le jacobinisme expliquerait l'attrait des Français pour le communisme au $x^{e}$ siècle.

Stigmatisée comme " proto-totalitaire », la référence politique à la Révolution ne s'en relèvera pas. En revanche, l'influence furétienne sur I'historiographierévolutionnaireestnettementplusdiscutable.Claire,vive et précise, I'analyse deChristofferson soulève doncun certain nombre de questions, par exemple sur les rapports entre l'antitotalitarisme, le révisionnisme et la contre-révolution, ou encore sur la réception d'un Furet « antitotalitaire » par les historiens américains spécialistes de la Révolution française, qui nécessitent un éclairage complémentaire. C'est pourquoi, dans le cadre de la rubrique « Regards croisés» des AHRF, nous avons souhaité donner la parole à Michael Scott Christofferson, en lui demandant de bien vouloir revenir sur les passages de son livre relatifs à I'historiographie de la Révolution française.

Julien LOUVRIER

$*$ 
Julien LOUVRIER

Pourriez-vous rappeler en quelques mots la genèse de votre travail ? Quel fut le point de départ de cette longue enquête surl'émergence et le déploiement de l'antitotalitarisme dans la vie intellectuelle et politique française de l'après-guerre?

\section{Michael Scott Christofferson}

Je m'intéresse depuis longtemps à l'année 1968 en France. J'ai écrit un mémoire de maîtrise sur la CFDT et l'autogestion. Mais j'ai commencé ma carrière $d$ 'historien en faisant une thèse de doctorat sur un autre sujet : I'histoire politique de la radiodiffusion en France dans les années 1930. Quand je suis arrivé en France en 1992 pour faire des recherches pour cette thèse, j'ai appris que Cécile Méadel venait de soutenir une thèse de doctorat d'État sur le même sujet. Comme sa thèse est très bien faite, mon sujet perdait de son intérêt. À cette époque, j'assistais comme auditeur libre au cours de Pierre Rosanvallon à I'EHESS. Rosanvallon y parlait souvent de l'importance de la critique du totalitarisme dans sa trajectoire intellectuelle et politique. Je me suis rendu compte qu'il y avait un sujet passionnant liéà mon intérêt premier pour l'année 1968. J'ai changé le sujet de ma thèse, et Robert Paxton, qui dirigeait ma thèse sur la radio, a très gentiment accepté de diriger cette nouvelle thèse quiétait un peu hors de sa grande expertise surles années 1930 et 1940 . Donc, je suis arrivé à ce sujet un peu par hasard.

Au niveau de la conceptualisation de ma problématique, mon enquête prend pourpoint dedépartleconstatqu'ilya undécalageimportantentrela chronologie du concept detotalitarismeen France et sa chronologie ailleurs. Les intellectuels français ont découvert le concept au milieu des années 1970, au moment même où le concept perdait une bonne partie de son influence aux États-Unis et en Allemagne de l'Ouest, ses pays d'origine. En plus, j'ai vite compris que la lecture des écrits antitotalitaires d'autres pays ne jouait aucun rôle dans la naissance de I'antitotalitarisme en France. Je suis arrivé à l'intuition que l'antitotalitarismefrançaisétaitdavantage un produit defacteursintérieursàlaFrance qu'une réponse aux développements extérieurs à l'hexagone. Suivant le fil de cette hypothèse, j'ai pu démontrer que L'Archipel du Goulag n'était pasla révélation quiavait déclenché une critique du totalitarisme.Non,ce n'est pas un choc extérieur qui a menéà l'antitotalitarisme, mais plutôt un conflitpolitiqueintérieur, notammentceluientrecertainsintellectuels de la gauche non communiste et l'Union de la gauche qui visait le pouvoir. 
Ce conflit explique à mon avis et la chronologie de l'antitotalitarisme et une bonne partie de son contenu.

\section{Julien LOUVRIER}

Dans un ouvrage intitulé Le marxisme et la gauche française ${ }^{2}$, l'his- $^{\prime}$ torien britannique Tony Judt suggère que dans la France contemporaine, la Révolution française a longtemps fait fonction de théorie politique, signifiant par-là que les intellectuels français auraient été plus occupés à se situer par rapportà la tradition révolutionnaire devenue une référence symboliquequ'àconstruire une science proprement politique.Votrelivre seconclut surl'offensive, provisoirement victorieuse, menée parFrançois Furet contre la lecture classique de la Révolution française au nom de la critique du totalitarisme. Il apparaît en fin de compte que l'antitotalitarisme fut le moyen le plus efficace que trouvèrent les intellectuels de la gauche non-communiste pour liquider la Révolution, à la fois comme référence et comme projet politique. Dès lors, en quoi la critique du totalitarisme dans la Révolution française se distingue-t-elle de la tradition contre-révolutionnaire?

\section{Michael Scott ChRISTOFFerSON}

Je répondrai en premier lieu à votre question en la déconstruisant. Si j'ai bien compris l'œuvre de Tony Judt sur ces questions, il est largement tributaire des aperçus de François Furet lui-même. La contribution de Judt est d'appliquer l'analyse de Furet du « catéchisme révolutionnaire $»^{3}$ à la gauche toute entière. Selon l'article célèbre de Furet, les historiens "jacobins» de la Révolution française ne la pensent pas; ils se contententderépéteruncatéchismerévolutionnaire.SelonJudt,lagauche ne pense pas la politique; elle se contente, elle aussi, d'un catéchisme. En plus, I'application pluslargedel'aperçudu « catéchismerévolutionnaire » est, en réalité, le point de départ de Furet lui-même. Depuis la fin des années 1960, Furet critiquait souvent l'incapacité de la gauche française à penser la politique, sauf en termes d'analogie historique ${ }^{4}$. Somme toute, cette question nous fait entrer dans un système auto-référant. Tout cela pour dire combien l'œuvre de Furet est étroitement liée à ses préoccupa-

(2) Tony JUDT, Le marxisme et la gauche française, Paris, Hachette, 1987, XIX-353 p.

(3) François FURET, «Le catéchisme révolutionnaire», Annales ESC, 26, 1971, p. $255-289$.

(4) Voir, par exemple, son article «France : la fin du parlementarisme », France observateur, 504, 30 décembre 1959, p. 9-10. 
tions politiques et combien la critique de cette œuvre est importante à la reconceptualisation non seulement del'historiographie de la Révolution française, mais aussi de l'histoire politique de la France contemporaine.

Pour répondre plus directement à votre question, je ne crois pas que Furet avait l'intention de suivre le sillon de la tradition contrerévolutionnaire. II croyait sincèrement que la Révolution de 1789 avait ouvert la voie à la démocratisation de la France. Contrairement aux contre-révolutionnaires, il ne voulait pas revenir en arrière en matière de démocratisation, mais plutôt apaiser la démocratie française en évacuant la révolution de la politique contemporaine. Comme les historiens allemands qui croyaient que leur pays suivait un Sonderweg (voie particulière), selon lequel la faiblesse de la tradition libérale, particulière à I'Allemagne, menait au nazisme, Furet croyait que la France suivait un Sonderweg à elle qui menait de "l'illibéralisme » proto-totalitaire de la Révolution française au totalitarisme de gauche du $x x^{\mathrm{e}}$ siècle. Tout le problème est que Furet insistait beaucoup plus sur la pente totalitaire de la Révolution que sur la contribution de la Révolution à la démocratisation, surtout dans son livre Penser la Révolution française ${ }^{5}$. Même s'il a essayé de rééquilibrer son interprétation plus tard, surtout après que de vrais contre-révolutionnaires, telquePierreChaunu,ontessayédes'approprier son œuvre,jecroisque lebilanfurétien dela Révolution resteplutôtnégatif. De plus, il faut se demander si la démocratie peut survivre sans faire appel à la tradition révolutionnaire à laquelle elle doit sa naissance. Je n'en suis pas sûr, en France du moins.

\section{Jacques Guilhaumou}

Vous considérez que François Furet introduit, dans ses travaux, une série de confusions peu propices au travail scientifique, confusion entre le travail historique et les préoccupations politiques, confusion entre l'historiographie et l'histoire, confusion entre la langue et la politique, c'est-à-dire entre la conscience révolutionnaire des acteurs et la réalité historique, et qu'il le fait à cause de son imprégnation permanente par la logique antitotalitaire. C'est donc d'une démarche jugée réductionniste que vous rendez compte. Est-ce que vous considérez que celainvalidevraimentsonapportscientifique, pourtantimportantdansle champ de l'histoire politique quand on voit son écho, en particulier chez les historiens américains?

(5) François Furet, Penser la Révolution française, Paris, Gallimard, 1978, 259 p. 


\section{Michael Scott ChRISTOFFERSON}

JedoiscommencerpardirequejesuishistoriendelaFrancecontemporaine et de l'historiographie révolutionnaire. Je ne suis pas historien de la Révolution de 1789. Donc, c'est moins à moi qu'aux historiens de la Révolution eux-mêmes de dresser le bilan de l'apport scientifique de l'œuvre de Furet. Ceci dit, j'ai quelques idées là-dessus. Je crois que Furet a fait une contribution très importante à l'histoire de la Révolution en tant que critique de l'historiographie telle qu'elle était au début des années 1970. Même si sa critique de l'historiographie qu'il a surnommée « jacobine » était très polémique, elle était souvent juste. En montrant les insuffisances des interprétations sociales de la Révolution et en insistant sur l'importance des dynamiques proprement politiques, il a joué un rôle central dans la renaissance de l'histoire politique de la Révolution. Il faut dire qu'il y avait eu avant lui d'autres historiens, tels le britannique Alfred Cobban et l'américain George Taylor, qui avaient montré certaines faiblesses de l'interprétation sociale de la Révolution, mais c'est Furet qui a faitéclater le consensus historiographique et a ouvert des voies nouvelles. Mais, si l'œuvre de Furet a de grands mérites iconoclastes, cela ne veut pas dire que l'interprétation qu'il propose soit à la hauteur de ses ambitions. Ses confusions entre le travail historique et les préoccupations politiques, entre l'historiographie et l'histoire, et entre la langue et la politique sont beaucoup plus présentes et importantes lorsqu'il essaie de bâtir une nouvelleinterprétation dela Révolutionquelorsqu'ilcritiquel'historiographie. L'apportscientifique de sa relecture dela Révolution mesemble beaucoup moindre que celui de son travail de déblayage de terrain.

Comme vous faites référence aux historiens américains, je donnerai quelques exemples d'outre-Atlantique pour illustrer mes propos. Même s'il y a quelques historiens américains assez proches de l'interprétation furétienne, tels Keith Baker et Patrice Higonnet, il y en a beaucoup d'autres sur lesquels Furet a exercé une grande influence, mais qui sont loin d'être furétiens. Par exemple, le livre phare de Lynn Hunt, Politics, Culture, and Class in the French Revolution ${ }^{6}$, a été écrit sous l'influence de Furet, mais Hunt n'a pas adopté son interprétation. Plus tard, elle a expliqué : "J'ai commencé par déclarer à un collègue français que je trouvais son analyse superficielle-une remarquequime metencore dans l'embarras-, mais je dois avouer que je me suis colletée avec elle pendant

(6) Lynn Hunt, Politics, Culture and Class in the French Revolution, Berkeley/Londres/Los Angeles, University of California Press, 1984, XV-251 p. 
un sacré bout de temps avant de finir par exorciser mon agitation dans un long compte rendu en 1981. En dépit de mon désaccord avec certains aspects de l'argumentation de Furet, je m'émerveillais de sa capacité à présenter les enjeux théoriques de l'événement. Le deuxième livre que j'ai écrit, Politique, culture et classe sociale dans la Révolution française, résulted'uneconfrontation directeavecson point devue $»^{7}$.Undeuxième exemple qui est peut-être révélateur, est celui d'Isser Woloch, avec qui j'ai étudié la Révolution française à Columbia University. Woloch insistait toujours surl'importance du « catéchisme révolutionnaire » de Furet, qui, selon lui, a ravivé une historiographie un peu complaisante. Mais, en même temps, Woloch a écrit une critique tranchante du Dictionnaire critique de la Révolution française de Furet et Mona Ozouf, que Furet n'a pas du tout appréciée ${ }^{8}$. Un autre exemple important est celui deTimothy Tackett. II a suivi Furet en s'investissant dans l'étude de la dynamique politique de la Révolution, mais son travail très empirique qui teste les conclusions plus spéculatives de Furet tend à les mettre en question?.

Donc, dans la mesure où Furet a modifié la problématique des historiens, oui, nous sommes tous ses héritiers et son apport scientifique est grand. Mais dans la mesure où son interprétation est discutable, son apport scientifique est moins important.

\section{Jacques Guilhaumou}

Quand le concept de « totalitarisme » s'est mis à occuper une place de plus en plus importante dans les débats politico-intellectuels qui agitaient la gauche française, il n'était plus usité aux États-Unis, et vous expliquez qu'Hannah Arendt elle-même y avait renoncé dès 1966. Vous montrez bien comment François Furet tout à la fois épouse et imprime sa marque à la lutte antitotalitaire en France pour produire une œuvre indissociable des enjeux dela politique contemporaine. En revanchevous ne documentez pas l'accueil favorable que Furet a pu rencontrer chez les historiens américains. Keith Baker et Patrice Higonnet, pour prendre les

(7) Lynn Hunt, "Fantasy Meets Reality: A Midwesterner Goes to Paris », in Laura Lee Downs and Stéphane GeRson, ed., Why France : American Historians Reflect on an Enduring Fascination, Ithaca, NY, Cornell University Press, 2007, p. 69.

(8) Isser Woloch, "On the Latent Illiberalism of the French Revolution », American Historical Review 95, 5, october 1990, p. 1452-1470.

(9) Timothy TACKETT, Becoming a Revolutionary: The Deputies of the French National Assembly and the Emergence of a Revolutionary Culture (1789-1790), Princeton, Princeton University Press, 1996; Idem, When the King Took Flight, Cambridge (Mass.), Harvard University Press, 2003; Idem, « Conspiracy Obsession in a Time of Revolution : French Elites and the Origins of the Terror, 1789-1792 », American Historical Review 105, 3, june 2000, p. 691-713. 
plus emblématiques des chercheurs américains qui se sont réclamés de Furet, sont-ils eux-aussi des historiens antitotalitaires ?

\section{Michael Scott ChRISTOFFerSON}

Il est vrai que je ne documente pas l'accueil de Furet aux ÉtatsUnis parce que mon livre est sur la critique du totalitarisme en général et ne consacre qu'un chapitre à Furet. Je n'ai pas étudié de très près l'œuvre de Baker ou celle de Higonnet, mais à première vue je ne crois pas qu'ils soient motivés par un antitotalitarisme quelconque. Baker, au moins, me semble plus influencé par le « tournant linguistique » que par des luttes proprement politiques. Mais, il ne faut pas sous-estimer l'importance de l'anticommunisme de la guerre froide outre-Atlantique. Un anticommunisme partagé pouvait servir de passerelle entre Furet et certains historiens américains, tel que George Taylor qui s'est défini comme historien chrétien de la Révolution ${ }^{10}$.

Votre question fait indirectement référence à un problème plus vaste. Je soutiens que la critique du totalitarisme des années 1970 en FranceestleproduitdesdéveloppementsinternesàlaFrance,notamment le débat surl'avenir dela gauche dans le contexte dela montée del'Union delagauche.En mêmetemps, il estincontestablequeFuretavait desliens importants avec des historiens américains. Si le concept de totalitarisme avait perdu de sa force auxÉtats-Unis pendantles années 1960 et nemotivait plus beaucoup d'historiens américains de la Révolution, comment expliquer leurs rapports avec Furet, d'ailleurs souvent très bons?

Du côté des historiens américains, il faut comprendre leurs relations avec Furet par rapport à leurs stratégies de consécration universitaire ${ }^{11}$.Entrelafindela deuxièmeguerremondialeetledébutdesannées 1970 , les historiens américains de la France avaient un complexe d'infériorité par rapport à leurs collègues français. Surtout dans les années 1950,beaucoupd'entre-euxnecroyaientpasqueleursrecherchesétaient à la hauteur de celles des Français qui avaient davantage accès aux archives. En même temps leurs livres n'étaient pas pris très au sérieux par les historiens français. Le premier livre de Robert Paxton sur Vichy a été passé sous silence. La Bibliothèque nationale de France n'a pas

(10) Voir à propos de Taylor, Peter Novick, That Noble Dream : The 'Objectivity Question' and the American Historical Profession, New York, Cambridge University Press, 1988, p. 408.

(11) J'ai l'intention d'étoffer cette analyse dans un article que je prépare. 
achetéle livre; Paxton a dû lui-même présenter un exemplaire ${ }^{12}$. Robert R. Palmer se plaignait du manque d'intérêt porté par les Français à son histoire des révolutions atlantiques de la fin du xville siècle et, par conséquent, est arrivé en 1970 à la conclusion que « peu de chercheurs universitairesdisposentd'uneorthodoxieaussigénéralementacceptéeque lesspécialistes dela Révolution française, confortablementétabliedans leurs Annales, une revue qui mentionne toujours fièrement sur sa couverture le nom de son fondateur, Albert Mathiez, mort il y a trente ans, et qui publie activement des recherches microscopiques qui ne défient aucunepenséeetquiredoutent toutautantles réinterprétationsqueles questions gênantes $»^{13}$.

Dans ce contexte Furet a joué un rôle très important pour les historiens américains. Invité assez régulièrement à séjourner dans les universités américaines à partir de 1968, Furet prenait au sérieux les historiens américains. Il s'appuyait sur leurs travaux sur les origines de la Révolution et sur la composition sociale des révolutionnaires pour critiquer l'orthodoxie historiographique. Il les aidait à s'orienter quand ils venaient en France pour faire leurs recherches. Il proposait la traduction de leurs livres, et il les invitait à la Ve section de l'EPHE et puis à I'EHESS pour donner des conférences et des séminaires. Beaucoup d'entre-eux n'étaient pas d'accord avec l'interprétation de la Révolution de Furet, et, pour la plupart, ils n'étaient pas à l'aise quand on les sommait de prendre partidans la guerre historiographique française, mais ils restaient reconnaissants à Furet de ce qu'il faisait pour leur réputation internationale.

\section{Julien LOUVRIER}

Faut-il faire des affrontements de Furet avec les historiens universitaires une polémique uniquement motivée par des stratégies politiques, comme si tout ce qui se manifestait sur le terrain de la science historique et dusavoir positifn'était que le symptôme dela lutteidéologique? L’ana-

(12) Robert O. Paxton, Parades and Politics at Vichy: The French Officer Corps under Marshall Pétain, Princeton, Princeton University Press, 1966; John F. SwEETs, « Chaque livre un événement : RobertPaxton and the French, from briseur de glace to iconoclaste tranquille », in Sarah Fishman, Laura Lee Downs, lonnis Sinanoglou, Leonard V. SMith, and Robert Zaretsky (eds.), France at War : Vichy and the Historians, New York, Berg, 2000, p. 21. Une traduction française du premier livre de Paxton (1966) a paru en 2004.

(13) Robert R. Palmer, "The Age of Democratic Revolution», in L. P. Curtis, ed., The Historian's Workshop: Original Essays by Sixteen Historians, New York, Alfred A. Knopf, 1970, p. 182-183. Voir du même, The Age of Democratic Revolutions, 2 vols, Princeton, Princeton University Press, 1959 et 1964. 
lysedescombatshistoriographiquesdanslechampdesétudesrévolutionnaires par la seule grille de lecture de l'antitotalitarisme ne minore-t-elle pas tout ce qui relève des effets de génération, de trajectoire, des choix individuels?

\section{Michael Scott ChRISTOFFerson}

Vous avez raison de dire que la lutte idéologique n'est pas le seul facteur qui explique l'histoire du combat historiographique. J'ai essayé de tenir compte d'autres facteurs en expliquant la dispute entre Furet et Soboul. Des différences de formation professionnelle, d'origines familiales, et d'assise institutionnelle ont joué des rôles importants. Une étude exhaustive aurait beaucoup plus à dire sur ces questions. Si j'ai concentré mon analyse sur l'antitotalitarisme, c'est, en partie, parce que mon livre se focalise sur l'antitotalitarisme et pas sur l'historiographie révolutionnaire. Mais, c'est aussi parce que je crois que la lutte idéologiqueétaitcentraleetdéterminantepourlaluttehistoriographique.Furet a pu gagner cette lutte idéologique dans les années 1970 et 1980 parce qu'il menait une bataille sur plusieurs fronts et sur plusieurs registres. Non satisfait de combattre ses adversaires dans les revues historiques françaises, il mobilisait les médias français et les historiens étrangers. Son argumentation ne restait ni au niveau de la science historique, ni même au niveau politique. II mettait en avant ses souvenirs d'excommuniste et valorisait l'ouverture institutionnelle de I'EHESS par rapport à l'université où Soboul avait le pouvoir. C'était une guerre totale.

\section{Julien LOUVRIER}

Votre manière de présenter la réception de Penser la Révolution française ${ }^{14}$ par les élites académiques qui s'expriment dans la presse quotidienne ou hebdomadaire à l'époque de la parution du livre, donne à penser que l'antitotalitarisme est un rouleau compresseur devant lequel aucune force n'a tenté de se dresser. N'aurait-il pas été juste de mentionnerquechezleshistoriensdelaRévolutionfrançaiselesthèsesfurétiennes furent loin de recevoir un accueil aussi unanime, c'est à dire replacer le travail de Furet dans le contexte universitaire auquel certes il échappait partiellement, mais auquelilne pouvaitmalgrétoutse soustraireentièrement? Dans les pages des Annales historiques de la Révolution française, 
I'historien Jacques Godechot, que l'on peut difficilement suspecter de philocommunisme, avait vivement réagi au livre qui proclamait «La Révolution est terminée » en se plaçant sur le terrain abandonné par Furet de la lutte contre le fascisme ${ }^{15}$. Son compte rendu mettait par ailleurs en cause le style d'écriture utilisé par Furet, qualifié par Godechot de « jargon à la mode chez les snobs parisiens »... indiquant par-là combien était grande la distance qui séparait les historiens de la Révolution française de tradition universitaire et le milieu dans lequel évoluait Furet.

\section{Michael Scott Christofferson}

Au début, Furet avait assez peu de soutien parmi les historiens universitaires de la Révolution en France. Comme vous l'avez montré, ces historiens ont soutenu Claude Mazauric dans la controverse autour de La Révolution française (1965-1966) de Furet et Richet. Les comptes rendus d'historiens favorables à ce livre sont venus exclusivement de leurs collègues de la V'e section de l'EPHE, Louis Bergeron et Marc Ferro $^{16}$. Pour gagner la lutte historiographique, Furet a dû trouver du soutien ailleurs que dansl'universitéfrançaise. Les historiens étrangers, surtout les historiens américains, y jouaient un rôle clé. Le rôle, expliquéplushaut, queFuretjouaitdanslesstratégies deconsécration universitaire des Américains n'était pas sans contrepartie. Furet avait besoin d'euxpourconstruireunecommunautéintellectuellealternativeetpour légitimersoninterprétationen dépitdel'oppositiond'unegrandepartie des universitaires français. Le rapport entreFuret et les historiens américains de la Révolution était symbiotique.

Pour justifier ce que j'avance, je vous donnerai un exemple basé sur mes recherches actuelles sur Furet et les historiens américains. Furet a fait une bonne partie du travail préparatoire à son article «le catéchisme révolutionnaire » quand il était à Princeton pendant l'année scolaire 1968-1969. Dans les archives personnelles de Furet au Centre de recherches politiques Raymond Aron, il y a trois commentaires sur le manuscrit de cet article. Ils sont de Louis Bergeron, de l'historien anglais

(15) « François Furet est trop jeune, sans doute, pour se souvenir que le régime de Vichy a voulu se venger non seulement du Front populaire, mais aussi de la Révolution. Les Droits de l'homme de 1789 ont été anéantis, la liberté et l'égalité jetés aux oubliettes [...] », AHRF, n²35, p. 135-141.

(16) Julien Louvrier, «Penser la controverse: la réception du livre de François Furet et Denis Richet, La Révolution française », AHRF, 351, 2008, p. 151-176. 
Richard Andrews, et de l'historien américain Robert Darnton. Sans allié dans l'université française, Furet a dû faire appel à ces jeunes historiens dont il a fait la connaissance à Princeton. Ce n'était que le début d'une stratégie d'internationalisation de l'historiographie révolutionnaire que Furet a utilisé à son très grand avantage contre ses adversaires historiographiques en France. Du Dictionnaire critique de la Révolution françaiseauxcolloquesinternationauxsurlaculturepolitiquedela Révolution française, Furet a fait appel à un monde plus vaste pour gagner la bataille historiographique en France.

Michael Scott CHRISTOFFERSON Associate Professor of History Penn State Erie msc8@psu.edu

Jacques GUILHAUMOU UMR «Triangle » Université de Lyon, CNRS/ENS-LSH 15 parvis René Descartes, BP 7000 69342 Lyon Cedex 07. France guilhaum@newsup.univ-mrs.fr

Julien LOUVRIER GRHIS - Université de Rouen Ohjaajantie 6 A 7 00400 Helsinki. Finlande Julien.louvrier@gmail.com 